Rabaska

Revue d'ethnologie de l'Amérique française

\title{
Chaire de recherche du Canada en oralité des francophonies minoritaires (COFRAM)
}

\section{Jean-Pierre Pichette}

Volume 5, 2007

URI : https://id.erudit.org/iderudit/019066ar

DOI : https://doi.org/10.7202/019066ar

Aller au sommaire du numéro

Éditeur(s)

Société québécoise d'ethnologie

ISSN

1703-7433 (imprimé)

1916-7350 (numérique)

Découvrir la revue

Citer ce document

Pichette, J.-P. (2007). Chaire de recherche du Canada en oralité des

francophonies minoritaires (COFRAM). Rabaska, 5, 222-226.

https://doi.org/10.7202/019066ar d'utilisation que vous pouvez consulter en ligne. 


\section{Rapports des institutions}

ACADIE

\section{Chaire de recherche du Canada en oralité des francophonies minoritaires (COFRAM)}

Centre acadien

Téléphone : (902) 769-2114

Université Sainte-Anne

Télécopieur : (902) 769-0063

Pointe-de-l'Église

(Nouvelle-Écosse) B0W 1M0

Courriel : jeanpierre.pichette@usainteanne.ca

Toile : www.usainteanne.ca

\section{Présentation}

La collaboration entre la Chaire de recherche en oralité des francophonies minoritaires (COFRAM) et le Groupe de recherche en études acadiennes (GRÉA) de l'Université Sainte-Anne a été cette année particulièrement intense. Leurs missions respectives coïncident d'ailleurs sur le plan du développement des recherches multidisciplinaires dans le vaste champ des études acadiennes. On pourra lire dans les deux numéros du bulletin du GRÉA le sommaire de leurs activités conjointes, notamment dans l'organisation et la conclusion de réunions scientifiques : accueil de chercheurs, de conférenciers, colloques, publication de la revue Port Acadie et lancement de divers ouvrages (GRÉA $\mathrm{n}^{\circ}$ 2, automne 2006, 2 p.; et GRÉA no 3, hiver 2007, 6 p. ; disponibles au site www.usainteanne.ca/documents/GREA_2.pdf et GREA_3.pdf).

\section{Réunions scientifiques}

A. Colloque international - Faisant suite à des échanges qui prirent naissance en 1991 et qui ont récemment mené aux premières Journées d'études de la chaire COFram à la Pointe-de-l'Église en octobre 2005 puis, en juin 2006, aux premières Rencontres britto-franco-canadiennes à Brest, les organisateurs ont convenu d'examiner le phénomène de la résistance des marges dans un colloque international en août 2007. Concerté par la CoFrAM et le GRÉA, avec la collaboration du Centre de recherche bretonne et celtique (CRBC, Université de Bretagne occidentale, Brest), de l'Unité de recherche en médiation culturelle (URMC, Université de Liège), leurs partenaires européens, et de la Société québécoise d'ethnologie (SQE, Québec), ce rendezvous rassemblera une quarantaine de chercheurs d'Europe, d'Afrique et 
d'Amérique qui réfléchiront autour du thème fédérateur de la Résistance des marges en fonction des axes suivants : exploration, transfert et revitalisation des traditions populaires des francophonies d'Europe et d'Amérique.

B. ÉDITION DES ACTES - Avec la planification de la rencontre internationale signalée ci-devant, c'est l'édition des actes des colloques antérieurs qui a retenu le plus clair de nos énergies à ce chapitre au cours de l'année 20062007. Ainsi, les actes du colloque national de juin 2006, le Patrimoine religieux de la Nouvelle-Écosse. Signes et paradoxes en Acadie sont maintenant sous presse et paraîtront comme numéro triple de la revue Port Acadie; de même, le compte rendu de la réunion de Rouen, à laquelle le titulaire a pris part, vient de paraître : Le Québec à la rencontre de la Normandie. Actes du colloque de l'AMOPA, 30-31 mai \& $1^{\text {er }}$ juin 2006. Textes réunis par Aurélien Boivin et Cécile Fouache, [s.l. Québec et Rouen], Aмоpa, [s.d. mai 2007], 143 p. Voir sa contribution en fin de document : J.-P. Pichette, « La Résistance des marges d'après les traditions des minorités françaises d'Amérique », p. [135]-142.

C. Chercheurs invités - En novembre 2006, la Cofram recevait deux chercheurs dans le cadre de ses activités conjointes avec le GrÉA. Ce fut d'abord M. Bernard Émont qui, le $1^{\text {er }}$ novembre, donna la troisième conférence de la CofRAm. Chargé de conférences à l'Université de Paris IV et spécialiste de la Nouvelle-France, le professeur traita d'une "gaillardise» de Marc Lescarbot dans un exposé fort documenté : «Lescarbot et la fascination de l'univers marin à travers le Théâtre de Neptune et l'ensemble de son œuvre ». Cette rencontre marquait le quatrième centenaire de la création de la première œuvre littéraire française d'Amérique, soit le Théâtre de Neptune en la Nouvelle-France, « représenté sur les flots de Port-Royal le quatorzième de novembre mille six cens six ». Le 22 suivant, M. Martin Fournier, coordonnateur, anima un atelier de présentation du projet d'Encyclopédie $d u$ patrimoine culturel de l'Amérique française. Partie d'une tournée de plusieurs centres universitaires acadiens, sa visite avait pour but de faire connaître la nature de ce projet et de répondre aux interrogations des partenaires en suscitant des collaborateurs.

Ensuite, du 11 au 15 juin 2007, le Centre acadien accueillit $\mathrm{M}^{\mathrm{me}}$ Patricia Heiniger-Casteret, ethnologue occitane de l'Université de Pau, qui inaugura un séjour de recherche au Canada par un stage au LABOR avec l'intention de développer une série de cours de maîtrise sur la production et l'analyse des sources orales, sur leur valorisation et le rapport avec le discours identitaire, et sur la sensibilisation au "patrimoine vivant». L'idée d'associer, autour 
des centres d'archives orales, les expériences franco-canadiennes reconnues dans les milieux scientifiques et les expériences françaises issues d'activités associatives est d'une très grande actualité en France à l'heure où des universités découvrent la documentation orale et que les centres d'ici ont déjà créé des protocoles particuliers pour le traitement de tels fonds. Intéressée aussi au phénomène des "néo-conteurs ", l'ethnologue en profita pour interroger les artisans du Festival de la parole de la baie Sainte-Marie.

\section{Laboratoire de littérature orale}

A. DÉPôTS - Une vingtaine de collections, conçues comme travail de recherche des étudiants d'ethnologie de l'Université Sainte-Anne, ont enrichi les archives du Laboratoire de littérature orale et, partant, celles du Centre acadien. Cette documentation, qui totalise 583 pièces (487 enregistrées et 96 manuscrites), se compose surtout de chansons populaires, de genres narratifs brefs (légendes, anecdotes, mystifications) et de formulettes diverses (superstitions, comptines, virelangues, sobriquets), et leurs aires d'enquête touchent plusieurs provinces : la Nouvelle-Écosse principalement, mais aussi le Nouveau-Brunswick et le Québec. Quelques autres fonds documentaires audiovisuels, réalisés par les services de notre technicien, ont aussi été versés au LABOR en cours d'année : ce sont les captations des deux premières éditions du Festival de la parole de la baie Sainte-Marie (2005 et 2006), une manifestation à laquelle la COFRAM est associée, qui forment un corpus de plusieurs dizaines d'heures.

B. Traitement des collections - Le traitement de la collection sonore du titulaire se poursuit au LABOR. Une équipe travaille toujours à la transcription préliminaire des enregistrements sonores de ce fonds et elle s'est concentrée sur les enquêtes menées au Québec et en Ontario. Les trois assistants assignés à cette tâche ont commencé à intégrer en les vérifiant les relevés effectués antérieurement puis à produire une première version écrite des pièces enregistrées. Parallèlement à cette activité essentielle, notre secrétaire a commencé à insérer ces premiers documents dans la banque informatisée de cette collection en vue de sa consultation éventuelle.

C. Guide D'ÉCoute - L'indexation de la collection J.-Alphonse-Deveau, entreprise au cours de l'été dernier, a retenu un membre de l'équipe durant toute l'année universitaire et devrait s'achever en 2007. L'outil de recherche qu'il prépare servira de modèle pour le traitement des autres collections sonores du Centre acadien et facilitera leur consultation puisque les fiches d'entrevues, dorénavant aussi complètes que les documents l'autorisent, seront munies de la liste chronométrique des sujets abordés. 
D. TERRAIN - En plus du travail des assistants de recherche au cours de la période estivale, une première mission de terrain, conduite à l'intérieur d'un cours sur la fête, s'est déroulée en mars 2007 : la « Mission Mi-Carême ». Centrée sur la tradition très vivante qui prend place d'année en année à SaintJoseph-du-Moine et à Chéticamp, dans l'Île-du-Cap-Breton, en dehors de la saison touristique, cette mission a passionné les étudiants inscrits qui, tout en participant véritablement à cette célébration traditionnelle, l'ont documentée par des moyens techniques actuels pour le bénéfice du LABOR et des chercheurs du Centre acadien. Ils ont été frappés par le rôle que paraît jouer ce rituel dans la reprise en main de l'activité économique de ces communautés affectées par l'effondrement de la pêche. L'équipe du LABOR prépare une exposition et un documentaire vidéo sur cette expérience.

\section{Publications}

A. RABASKA - Le quatrième volume de Rabaska, revue d'ethnologie de l'Amérique française, a paru à l'automne 2006 (Québec, SQE, vol. 4, 2006, 267 p.) et il a été lancé à Québec en décembre. Produite par la CoFram, cette publication de la Société québécoise d'ethnologie unit en partenariat les universités de Moncton (Ronald Labelle), du Québec à Montréal (Yves Bergeron), de Régina (Dominique Sarny) et, bien entendu, la SQE (Jean Simard) autour de l'Université Sainte-Anne (J.-P. Pichette). Les textes d'une cinquantaine d'auteurs composent la dizaine de rubriques et de subdivisions habituelles de cet annuel unique en son genre. En parcourant le volume, on notera le travail du personnel du laboratoire : titulaire, secrétaire et assistants de recherche.

B. CAHIERs CharLeVoIX - Collectif voué à l'étude de l'Ontario français, la Société Charlevoix est présentement formée de neuf universitaires qui se rencontrent deux fois l'an pour discuter des travaux de recherche qu'ils consacrent à l'Ontario français et qu'ils diffusent ensuite dans les Cahiers Charlevoix. En 2006, ils ont publié le septième volume de cette série : Cahiers Charlevoix 7. Études franco-ontariennes (Sudbury, Société Charlevoix et Prise de parole, 2006, 335 p.). Outre l' « Avant-Propos » (p. 5-12) qu'il signe à titre de rédacteur depuis le premier cahier, J.-P. Pichette y donne aussi une longue étude : «Le Chercheur de trésors ou l'influence d'un livre. Marius Barbeau et le Romancero du Canada» (p. 85-143), portant sur l'un des ouvrages ethnologiques les plus marquants de l'Ontario français. Les autres coauteurs de ce volume sont: Gaétan Gervais, Yves Frenette, Simon Laflamme, Raymond Mougeon, Michel Bock et Michel Gaulin. 
C. Autres - Le titulaire de la Cofram a préparé les analyses et l'index des types des vingt-six contes du beau recueil de Joseph-Médard Carrière, une publication posthume préparée à l'Université de Sudbury : Contes du Détroit recueillis par Joseph-Médard Carrière, présentés par Marcel Bénéteau et Donald Deschênes (Sudbury, Prise de parole, «Agora », 2005). Pour plus de détails, on consultera La Mouvée, bulletin d'information de la Cofram, $\mathrm{n}^{\circ} 2$, décembre 2006, 8 p., qui est disponible au site www.usainteanne.ca/ documents/la_mouvee_2.pdf.

JeAn-Pierre Pichette

\section{Chaire de recherche McCain en ethnologie acadienne (CREMEA)}

Département d'Études françaises

Université de Moncton

Téléphone : (506) 858-4592

Moncton (Nouveau-Brunswick) Courriel : ronald.labelle@umoncton.ca E1A 3E9

La Chaire de recherche McCain en ethnologie acadienne (CREMEA) a été créée en 2006 grâce à un don généreux de la Fondation McCain. Elle a comme mandat de participer à la formation des étudiants au département d'Études françaises en dispensant des cours sur l'ethnologie acadienne, de contribuer aux activités de recherche menées au sein du département, de former des équipes de chercheurs afin de faire avancer la recherche tant sur la culture acadienne traditionnelle que sur des phénomènes ethnologiques contemporains, de mettre en valeur les ressources patrimoniales acadiennes par la réalisation d'ouvrages destinés à un public varié et d'assurer une présence de l'Université de Moncton dans le domaine des études ethnologiques tant au Canada qu'à l'étranger.

\section{Programmes principaux}

La CREMEA a obtenu en mars 2007 une subvention de 29000 \$ du CRSH dans le cadre de son Programme de recherche et de diffusion lié aux langues officielles. Le projet s'intitule «Mise en valeur des ressources culturelles franco-terreneuviennes ». Ce projet vise à produire trois types de ressources documentaires : une trousse à vocation pédagogique comprenant des documents sonores sur disques compacts accompagnés de transcriptions, un recueil de chansons folkloriques accompagné d'illustrations sonores, et finalement un site électronique consacré à la culture traditionnelle francoterreneuvienne. 